\title{
Searches for Dark Sector Mediators in the DUNE Multi-Purpose Near Detector
}

Jeffrey M. Berryman, André de Gouvêa, Patrick J. Fox, Boris J. Kayser, Kevin J. Kelly, and Jennifer L. Raaf

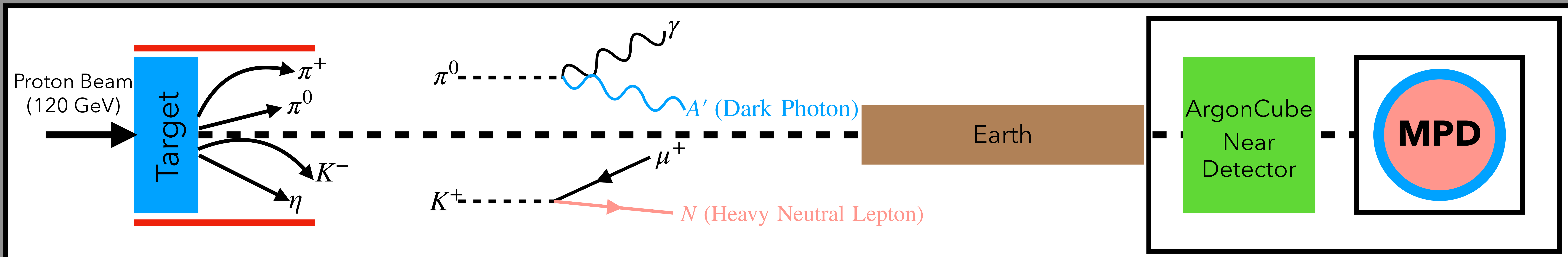

Magnetic Focusing Horns

Step 1: Charged and Neutral Mesons are produced in the high-energy proton collisions on the DUNE Target.

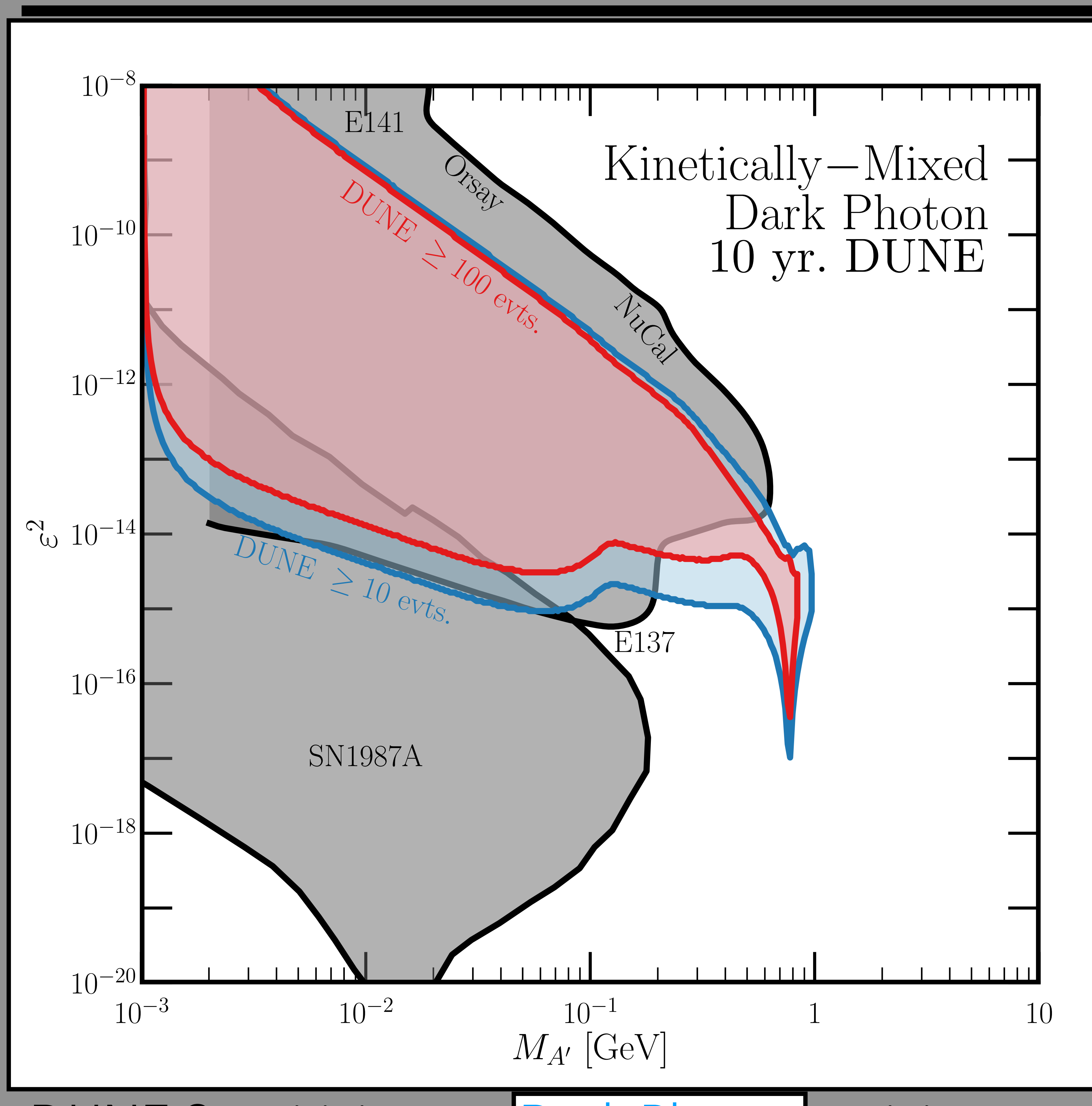

DUNE Sensitivity to a Dark Photon, mixing kinetically with the standard model. The blue and red regions indicate where DUNE can improve on existing constraints, shown in grey.
Decay Volume

Step 2: Mesons undergo rare decays into new, dark sector mediators that are relatively longlived. These new particles then can travel to the DUNE Near Detector Complex.
DUNE Near Detector Complex

Step 3: New physics particles decay into standard model particles, leaving signatures that are difficult for neutrino interactions to fake.

One of the most puzzling mysteries of physics is the overwhelming evidence for the existence of dark matter - from measurements of the cosmic microwave background to galactic rotation curves and beyond. Other than the fact that it interacts with standard model particles via gravity, no other interaction has been measured.

Many dark sector theories suggest that mediator particles allow for communication between the standard model particles and one or more dark matter particles. These mediators come in a variety of types, among them dark photons, dark Higgs bosons, Leptophilic gauge bosons, and

Depending on their masses, these new physics particles can be produced in the decays of charged and neutral mesons in a neutrino experiment's target area. We explore the possibility of searching for these particles using the Deep Underground Neutrino Experiment's Multi-Purpose Near Detector (MPD), a gaseous argon time-projection chamber embedded in an electromagnetic calorimeter and and a magnetic field. With ten years of data collection, DUNE will be able to probe a wide region of currently-unexplored parameter space in many different dark sector models.

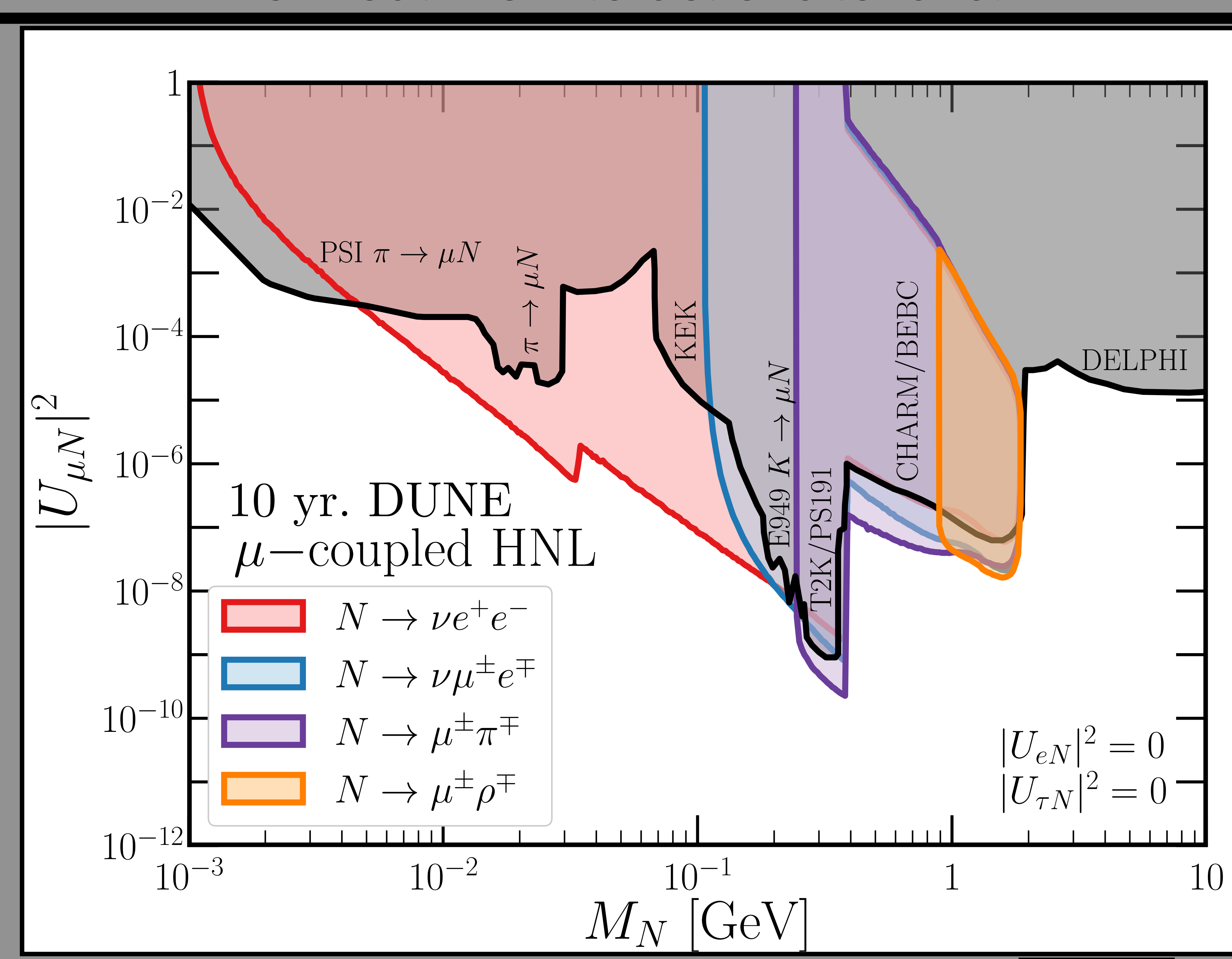

DUNE MPD Sensitivity to a muon-coupled [ Colored regions correspond to different final-state searches at DUNE. Grey regions correspond to existing constraints on this scenario.

Existing limits can be improved by as many as three orders of magnitude with ten years of DUNE data.

Work based on JHEP 02 (2020) 174 supported by DOE Awards DE-SC0018327, DE-SC0020262, DE-SC0010143, DE-AC02-07CH11359. 